\title{
Brief
}

\section{Partial atrioventricular canal defect with cor triatriatum sinister: Report of three cases}

Praveen K. Varma, MCh, Girish Warrier, MCh, Padmakumar Ramachandran, DM, Praveen Kumar Neema, MD, Soman Rema Krishna Manohar, MCh, Thomas Titus, DM, and Kurur Sankaran Neelakandhan, MCh, Trivandrum, India

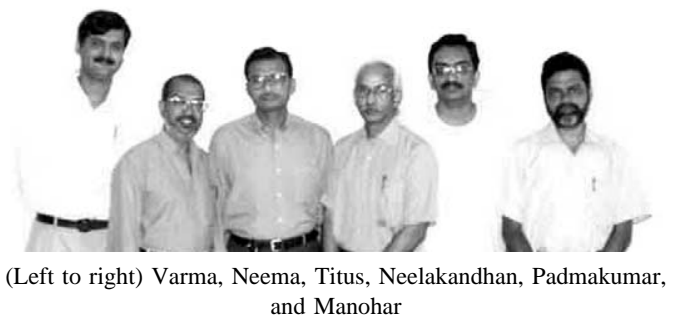
and Manohar
From the Departments of Cardiology and Cardio Vascular and Thoracic Surgery, Sree Chitra Tirunal Institute for Medical Sciences and Technology, Trivandrum, India.

Received for publication Jan 10, 2003; revisions requested Jan 21, 2003; revisions received Jan 26, 2003; accepted for publication March 25, 2003.

Address for reprints: Praveen K. Varma, MCh, Assistant Professor, Department of Cardio Vascular and Thoracic Surgery, Sree Chitra Tirunal Institute for Medical Sciences and Technology, Trivandrum, Kerala, India $695011 \quad$ (E-mail: pkvarma@sctimst.ker.nic.in).

J Thorac Cardiovasc Surg 2004;127:572-3

$0022-5223 / \$ 30.00$

Copyright ( $\odot 2004$ by The American Association for Thoracic Surgery

doi:10.1016/S0022-5223(03)01029-8

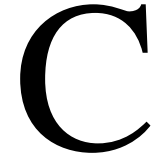

or triatriatum is an uncommon but surgically correctable cause of pulmonary venous hypertension and congestive cardiac failure, with a reported incidence of $0.1 \%$ among children with congenital heart diseases. Association with partial atrioventricular canal defect (PAVCD) is even rarer, with only anecdotal reports appearing in the literature. In the classic form, cor triatriatum is characterized by the presence of a fibromuscular diaphragm that subdivides the left atrium into a proximal accessory chamber and a distal true chamber.

\section{Clinical Summaries}

PATIENT 1. A 14-year-old boy was seen with class 2 exertional dyspnea. Clinical examination revealed fixed splitting of the second heart sound in the pulmonary area and short systolic murmur (grade 3/6) at the apex. Chest radiography showed right atrial enlargement and plethoric lung fields. Transthoracic echocardiography (TTE) demonstrated the presence of PAVCD with moderate left atrioventricular valve regurgitation and a dilated coronary sinus, suggestive of left superior vena cava. PAVCD was repaired under cardiopulmonary bypass. Postoperative TTE showed turbulence in the supramitral area with suspected membrane positioned obliquely and superiorly within the left atrium. Angiocardiography demonstrated an hourglass-shaped left atrium because of cor triatriatum (Figure 1, $A$ and $B$ ). The pulmonary artery wedge pressure was elevated (16-22 mm Hg, mean $16 \mathrm{~mm} \mathrm{Hg}$ ), with a gradient of $11 \mathrm{~mm} \mathrm{Hg}$ from the left ventricular end-diastolic pressure.

PATIENT 2. A 4-year-old girl was evaluated for recurrent respiratory tract infection. TTE revealed PAVCD with mild left atrioventricular valve regurgitation and a patent ductus arteriosus. During surgery, a diaphragm separating the left atrium in to a proximal pulmonary venous chamber and a distal true chamber with a $7-\mathrm{mm}$ central communication was identified. The left atrial appendage was arising from the distal chamber. The membrane was excised, and the PAVCD was repaired successfully.

PATIENT 3. An 18-year-old woman had PAVCD with moderate left atrioventricular valve regurgitation diagnosed by TTE. During the operation, while the surgeon was checking for the orifices of pulmonary veins, a thin diaphragm partitioning the atrium with a central hole of $12 \mathrm{~mm}$ was found. The coronary sinus was unroofed. The proximal chamber was communicating to right atrium by a stretched foramen ovale. The appendage was arising from the distal true chamber. The membrane was excised, and the rest of the repair was carried out uneventfully.

\section{Discussion}

These cases illustrate the importance of looking for associated lesions while managing congenital cardiac defects. The clinical picture of cor triatriatum depends on the size of the communication in the obstructing membrane ${ }^{1}$ and the associated intracardiac defects. Fea- 

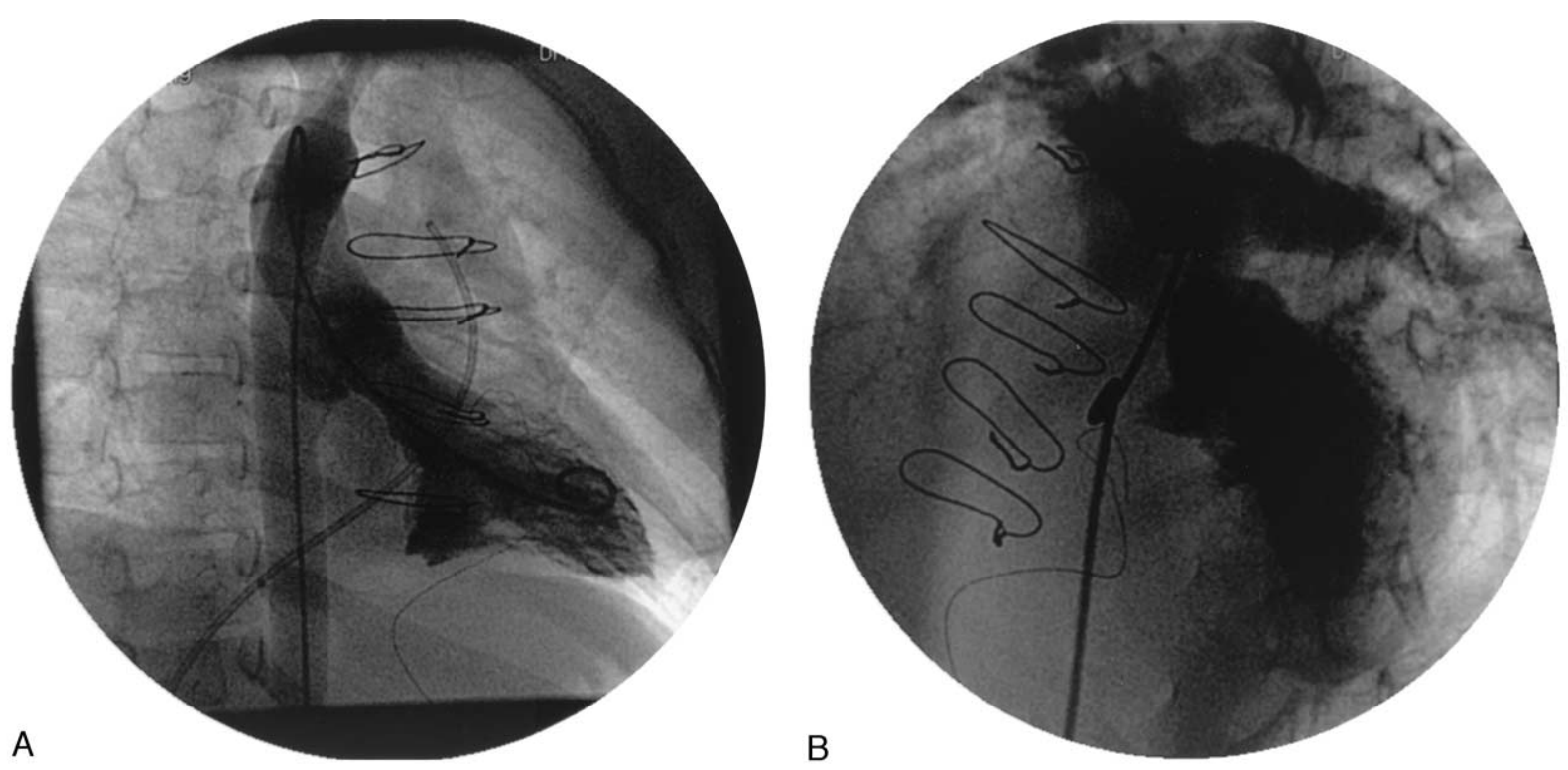

Figure 1. A, Pulmonary artery injection in levo phase shows all pulmonary veins draining into proximal chamber separated from distal chamber by hourglass constriction. Hourglass configuration is rare and usually associated with complex defects. B, Left ventriculogram shows classic gooseneck deformity of PAVCD; there is no regurgitation of atrioventricular valve.

tures of severe pulmonary venous hypertension dominate the clinical picture when the orifice is smaller than $3 \mathrm{~mm},{ }^{1}$ whereas these symptoms are mild when the orifice is larger than $7 \mathrm{~mm} .{ }^{1}$ Association of cor triatriatum with atrioventricular canal defects is rare; only 3 cases with complete atrioventricular canal and 7 cases with partial defect have been reported. ${ }^{2}$ Three of these cases are reported from autopsy specimens, with the defect's existence missed during life. ${ }^{2-4}$ The first case was reported by Gahagan and Ziegler ${ }^{3}$ in 1965, in which cor triatriatum was missed during surgery, leading to postoperative death and subsequent diagnosis at autopsy. Marin-Garcia and colleagues ${ }^{4}$ have reported 2 more cases of this combination found after autopsy, but both patients had not undergone operation. A major difficulty encountered in diagnosing left atrial membrane when associated with another cardiac lesion is that pulmonary venous obstruction is frequently masked. ${ }^{5}$ This scenario is unfortunate, because this is one of the easily correctable causes of pulmonary venous obstruction leading to pulmonary artery hypertension and congestive failure.

Retrospectively, we reviewed the preoperative TTE pictures. There was not enough evidence to suspect cor triatriatum in these patients. In shunt lesions at the atrial level, because of volume overload of right-sided chambers of the heart, there is clockwise rotation of heart and the left atrium and the left ventricle is pushed posterior. Therefore in the apical four-chamber view the left atrium is foreshortened, and thus associated cor triatriatum may be missed. Moreover, the left atrioventricular valve regurgitation and the shunt masked the presence of any gradients. This fact is further amplified by the first case report, where despite the poor echocardiographic window after the operation the membrane was identi- fied by TTE. This was because of correction of volume overload and the atrioventricular valve regurgitation.

In the era of correction of congenital anomalies diagnosed by TTE evidence only, this combination may come as diagnostic surprise at the operating table. Angiocardiography need not be diagnostic and is invasive. Marin-Garcia and colleagues ${ }^{4}$ could not clearly distinguish a left atrial membrane in any of the 4 patients catheterized, and Jacobstein and Hirschfeld ${ }^{5}$ could detect it in only 1 of 4 patients. During angiocardiography, the stasis in the proximal chamber suggesting obstruction may be overlooked, and the negative shadow cast by the membrane is unimpressive. Cardiac magnetic resonance imaging promises to be a useful tool in diagnosing anomalies of pulmonary connection and drainage, but it is expensive. Thus the operating surgeon should detect and correct this uncommon anomaly.

\section{References}

1. Niwayama G. Cor triatriatum. Am Heart J. 1960;59:291-317.

2. Goel AK, Saxena A, Kothari SS. Atrioventricular septal defect with cor triatriatum: case report and review of the literature. Pediatr Cardiol. 1998;19:243-5.

3. Gahagan T, Zeigler RF. Triatrial heart with persistent ostium primum and cleft mitral valve. Ann Thorac Surg. 1967;3:231-4.

4. Marin-Garcia J, Tandon R, Lucas RV Jr, Edwards JE. Cor triatriatum: study of 20 cases. Am J Cardiol. 1975;35:59-66.

5. Jacobstein MD, Hirschfeld SS. Concealed left atrial membrane: pitfalls in the diagnosis of cor triatriatum and supravalve mitral ring. Am $J$ Cardiol. 1982;49:780-6. 